\title{
A Biomathematical Modeling Approach to Central Nervous System Radioligand Discovery and Development
}

\author{
Qi Guo ${ }^{1}$, Michael Brady ${ }^{1}$, and Roger N. Gunn ${ }^{1-3}$ \\ ${ }^{1}$ Department of Engineering Science, University of Oxford, Oxford, United Kingdom; ${ }^{2}$ GlaxoSmithKline, Clinical Imaging Centre, \\ Hammersmith Hospital, London, United Kingdom; and ${ }^{3}$ Division of Neuroscience and Mental Health, Imperial College, London, \\ United Kingdom
}

\begin{abstract}
The development of a successful PET or SPECT molecular imaging probe is a complex, time-consuming, and expensive process that suffers from high attrition. To address this problem, we have developed a biomathematical modeling approach that aims to predict the in vivo performance of radioligands directly from in silico/in vitro data. Methods: The method estimates the in vivo nondisplaceable and total uptake of a ligand in a target tissue using a standard input function and a 1-tissue-compartment model with a parsimonious parameter set (influx rate constant $K_{1}$, efflux rate constant $k_{2}$, and binding potential $B P_{N D}$ ) whose values are predicted from in silico/in vitro data including lipophilicity, molecular volume, free fraction in plasma and tissue, target density, affinity, perfusion, capillary surface area, and apparent aqueous volume in plasma and tissue. The coefficient of variation of the $B P_{N D}\left(\% \operatorname{COV}\left[B P_{N D}\right]\right)$ metric, derived from Monte Carlo simulations, is used to estimate the in vivo performance of candidate compounds. A total of 28 compounds for 10 targets was evaluated using our method to predict their in vivo performance and validated against measured in vivo PET data in the Yorkshire/ Danish Landrace pig. Results: The predicted $K_{1}, k_{2}$, and $B P_{N D}$ values were generally consistent with the values estimated from in vivo PET data. The model resulted in small \%COV $\left[B P_{N D}\right]$ values for widely accepted good ligands such as ${ }^{11} \mathrm{C}$-flumazenil (2.02\%) and ${ }^{11} \mathrm{C}$-raclopride $(2.55 \%)$, whereas higher values resulted from poor ligands such as ${ }^{11} \mathrm{C}-(R)-\mathrm{PK} 11195$ (13.34\%). Of 4 candidates for the GlyT1 transporter, the model selected ${ }^{11}$ C-GSK931145 $(2.11 \%)$ as the most promising ligand, which was consistent with historical decisions made on the in vivo PET data. Conclusion: A biomathematical modeling approach has the potential to predict the in vivo performance of ligands from in silico/in vitro data and aid in the development of molecular imaging probes.
\end{abstract}

Key Words: positron emission tomography; imaging; radioligand; biomathematical modeling; radiochemistry

J Nucl Med 2009; 50:1715-1723

DOI: 10.2967/jnumed.109.063800

Received Mar. 4, 2009; revision accepted May 21, 2009.

For correspondence or reprints contact: Roger N. Gunn, GlaxoSmithKline, Clinical Imaging Centre, Hammersmith Hospital, Du Cane Rd., London, W12 ONN, U.K.

E-mail: roger.n.gunn@gsk.com

COPYRIGHT $\odot 2009$ by the Society of Nuclear Medicine, Inc.
M olecular imaging of the central nervous system (CNS) with PET and SPECT is predicated on the availability of radioligands that bind to a particular receptor, transporter, or enzyme of interest. A successful in vivo imaging probe possesses a range of properties including high selectivity for the target of interest, the ability to be safely administered to humans at tracer doses, the ability to reach the target site, low nonspecific binding, suitable affinity such that a large enough specific-tononspecific signal exists, suitably reversible kinetics to facilitate quantitative analysis, and the ability to be radiolabeled. Given these numerous and sometimes conflicting characteristics, it is not surprising that the discovery and development process is challenging (1).

To date, the process usually concentrates on screening compounds according to lipophilicity, affinity, selectivity over assumed nearest pharmacology, target density, and labeling feasibility. Lipophilicity, estimated as $\log D$, is assessed to determine the likelihood that a molecule will penetrate the blood-brain barrier (BBB); as a rule of thumb a value between 1 and 3 is chosen to maximize penetration while trying to minimize nonspecific binding (2). Compound affinity is used to determine whether the molecule binds avidly enough to the target to produce a measurable signal. The weakness of these approaches is that only some of the characteristics required are considered, with little deliberation given to the prediction of nonspecific binding and optimal kinetics. Even for the properties that are considered, there is little quantitative assessment of their likely impact on the in vivo outcome measures of interest. More recently, Patel et al. (3) have developed an in vitro nowash autoradiography assay to predict whether a candidate compound has a suitable specific signal. However, the authors acknowledged that this assay is still somewhat simplistic and does not consider brain entry or the kinetics of the compound.

Currently, even with in silico/in vitro screening of putative tracers, followed by preclinical evaluation and translation to humans, the process is expensive and time-consuming and 
suffers from a large amount of attrition. Thus, the discovery and development of novel radioligands is still essentially progressing by trial and error, with success often based on empiricism and serendipity (4). Fowler (5) also highlighted this in a commentary in The Journal of Nuclear Medicine and suggested that more sophisticated approaches to prediction are required: "Poor predictability has been a root cause of the low yield of useful radiotracers relative to the number of labeled compounds that have been painstakingly synthesized and evaluated over the years."

In this article, we introduce a biomathematical modeling approach that takes known physicochemical and pharmacological properties of candidate molecules to predict their in vivo performance. The idea was to develop a quantitative in silico method as a precursor to in vivo studies, aiming to provide increased confidence in candidate molecules before radiolabeling. Such an in silico screening tool would be ideally positioned to leverage large databases of compounds available in the pharmaceutical industry.

The biomathematical model incorporated quantitative components accounting for BBB penetration, specific binding, nonspecific binding, and radiotracer kinetics. These components were integrated within a tracer compartmental framework and parameterized using in silico/in vitro data enabling the simulation of in vivo datasets and the prediction of the outcome measure of interest. Here, we used the binding potential $\left(B P_{N D}\right)$ as the outcome measure, which is defined, at equilibrium, as the ratio of specifically bound to nondisplaceable radioligand in tissue (6). Our approach estimated the $B P_{N D}$ through Monte Carlo simulations and determined its magnitude and reproducibility metric as a measure of in vivo performance. Validation of the approach was investigated with a dataset of candidate radioligands $(n=28)$ for which there exists in silico/in vitro data and in vivo PET data acquired in the Yorkshire/Danish Landrace pig.

\section{MATERIALS AND METHODS}

The biomathematical modeling approach uses a 1-tissue-compartment model (1TCM) parameterized by in silico/in vitro data to predict the in vivo behavior of a radioligand (Fig. 1). The in vivo performance of candidate compounds was assessed through Monte Carlo simulations using the coefficient of variation of $B P_{N D}\left(\% \operatorname{COV}\left[B P_{N D}\right]\right)$ metric. An in silico/in vitro/in vivo dataset was introduced, and the validation of the individual model components and their overall performance was investigated.

\section{The Biomathematical Model}

The approach uses a 1TCM to approximate both the nondisplaceable and total uptake of a ligand in a target tissue. Thus, the assumption was that all compartments equilibrate rapidly. Though this may not always be the case, given the overall complexity of in vivo prediction, the assumption was reasonable and allowed for a parsimonious description in terms of just 3 parameters (influx rate constant $K_{1}$, efflux rate constant $k_{2}$, and $\left.B P_{N D}\right)$. These parameters were predicted from in silico/in vitro data to account for brain entry, specific or nonspecific binding, and kinetics. In conjunction with an appropriate plasma input function, the in vivo kinetics can be simulated and the behavior of the radioligand can be characterized.
FIGURE 1. Overview of the biomathematical modeling approach.

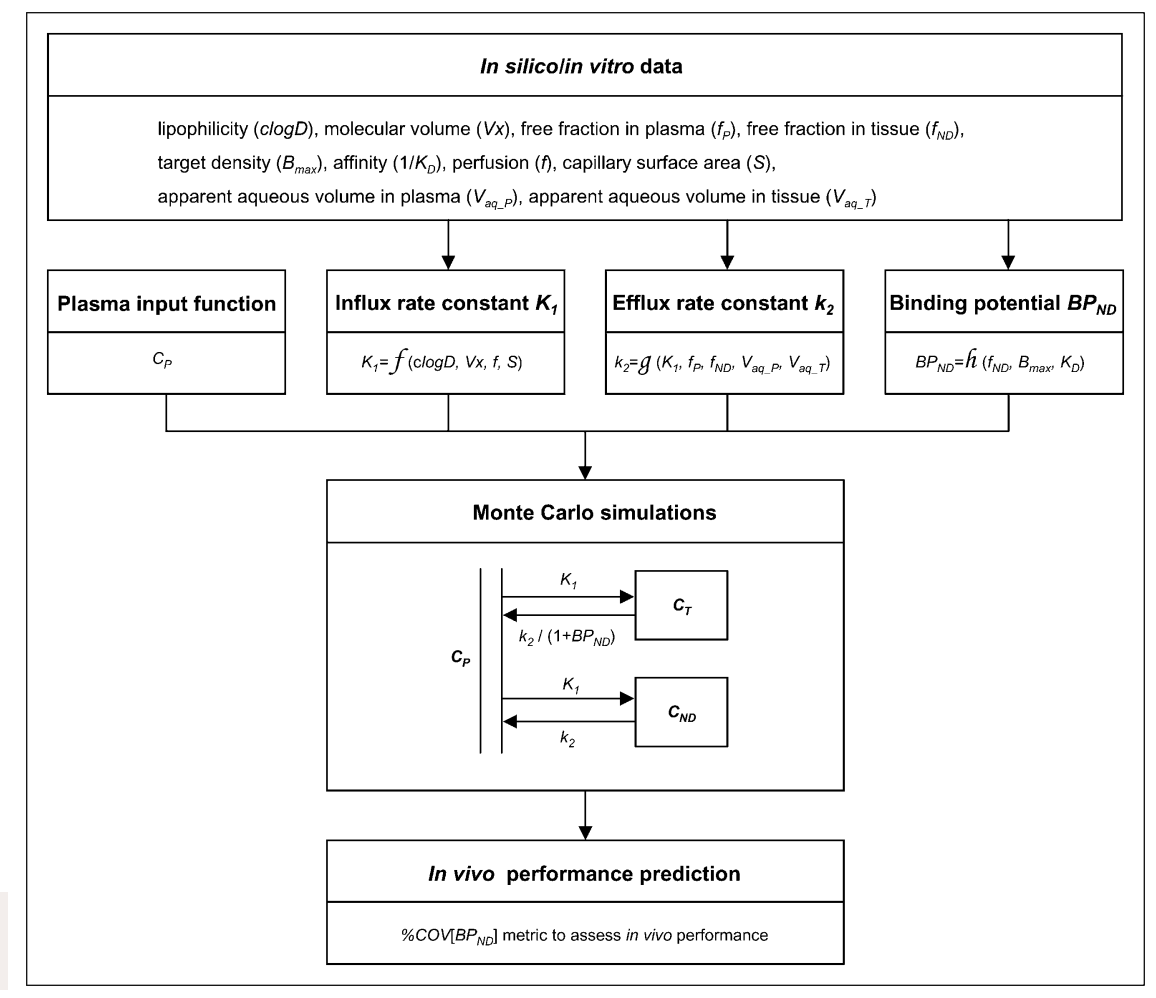


The $B P_{N D}$ was chosen as the primary outcome measure of interest, and the in vivo performance of the ligand was determined from its $\% \operatorname{COV}\left[B P_{N D}\right]$.

To approximate the in vivo behavior of the ligand, the $K_{l}, k_{2}$, and $B P_{N D}$ parameters predicted from in silico/in vitro data were used in conjunction with the 1TCM to generate noiseless timeactivity curves for $90 \mathrm{~min}$. Subsequently, 1,000 noisy realizations were generated with the variance of noise proportional to radioactivity divided by frame duration, and the 1TCM was fitted to both the nondisplaceable and the total tissue data to derive estimates of $B P_{N D}$ as follows:

$$
B P_{N D}=\frac{V_{T}}{V_{N D}}-1
$$

where $V_{T}$ and $V_{N D}$ are the volumes of distribution in the total and nondisplaceable compartments, respectively. This Monte Carlo simulation approach allows for the calculation of $\% \operatorname{COV}\left[B P_{N D}\right]$ :

$$
\% \operatorname{COV}\left[B P_{N D}\right]=\frac{\sigma\left(B P_{N D}\right)}{\mu\left(B P_{N D}\right)} \times 100 \%, \quad \text { Eq. } 2
$$

where $\sigma\left(B P_{N D}\right)$ is the $\mathrm{SD}$, and $\mu\left(B P_{N D}\right)$ is the mean of the $B P_{N D}$ estimates derived from the simulations. This metric is used to assess radioligand performance, with a smaller \%COV $\left[B P_{N D}\right]$ value indicating a better radioligand.

Models for the prediction of the parameter set $\left(K_{1}, k_{2}\right.$, and $B P_{N D}$ ) were developed using the physicochemical and pharmacological properties of the ligand, the target density, and additional physiological parameters.

\section{Prediction of $K_{1}$}

The influx rate constant $K_{l}$ gives an index of the ease with which a compound can transfer from plasma into the brain. Here, the model developed by Renkin and Crone $(7,8)$ is used to predict the brain uptake, which defined $K_{I}$ in terms of perfusion $(f)$, permeability of the compound $(P)$, and capillary surface area $(S)$ using the following equation:

$$
K_{1}=f\left(1-e^{-\frac{P S}{f}}\right)
$$

where $K_{l}$ and $f$ are in units of $\mathrm{mL} / \mathrm{cm}^{3} / \mathrm{min}, P$ is $\mathrm{cm} / \mathrm{min}$, and $S$ is $\mathrm{cm}^{2} / \mathrm{cm}^{3}$ of brain. In this model, $f$ and $S$ are known physiological parameters $(7,9)$. The permeability is the key property that determines the delivery of individual compounds across the BBB. We have used an in silico model to predict the permeability of candidate molecules from physicochemical properties (lipophilicity and molecular volume), assuming that the transport mechanism is passive diffusion. The model is a simplification of the permeability prediction model developed by Lanevskij et al. (10) and removes the flow limit and hydrogen bonding terms, combines ionization with lipophilicity using $\operatorname{cog} D$ (in silico logarithm of the octanol-water partition coefficient), keeps the McGowan volume term $V x$ (the actual volume of a mole when the molecules are not in motion $\left[\mathrm{cm}^{3} \mathrm{~mol}^{-1 / 100]}(11)\right)$, and replaces the bilinear function with a parabola. The model predicts the permeability as follows:

$$
P=10^{-0.121(c \log D-2.298)^{2}-2.544 \log \left(V x^{1 / 3}\right)-2.525} \text {. }
$$

The coefficient estimates of the model were determined using multilinear regression based on in vitro permeability data from 30
non-P-glycoprotein substrates, which were measured using the MDCK-mdr1 cell line (Madin-Darby canine kidney transfected with the multidrug resistance gene) (12). The model defines a parabolic relationship between permeability and lipophilicity and indicates an optimal $\operatorname{clog} D$ value of $2.3\left(R^{2}=0.58, P<0.0001\right)$.

\section{Prediction of $k_{2}$}

At equilibrium, under the assumption of passive diffusion, the free concentration of the compound will be the same either side of the BBB. The efflux rate constant $k_{2}$ can then be predicted from the influx rate $K_{l}$, the free fraction in plasma $f_{P}$, and the free fraction in tissue $f_{N D}$, which are, respectively, the fraction of unbound ligand in plasma and tissue that are free for binding. $f_{P}$ and $f_{N D}$ can be measured in vitro using equilibrium dialysis (12). The model also incorporates the apparent aqueous volume in plasma $V_{a q_{-} P}$ and in tissue $V_{a q_{-} T}$ to correct for the small volume that cannot be reached by solvent in plasma and tissue, respectively. Thus, it follows that at equilibrium,

$$
\frac{f_{P} \cdot C_{P}}{V_{a q \_} P}=\frac{f_{N D} \cdot C_{N D}}{V_{a q_{-} T}}
$$

where $C_{P}$ and $C_{N D}$ are the concentrations in plasma and nondisplaceable compartments, respectively. The volume of distribution of the nondisplaceable compartment is defined as follows:

$$
V_{N D}=\frac{C_{N D}}{C_{P}}=\frac{K_{1}}{k_{2}}
$$

Thus, $k_{2}$ can be predicted as:

$$
k_{2}=\frac{V_{a q_{-} P} \cdot K_{1}}{V_{a q_{-} T}} \cdot \frac{f_{N D}}{f_{P}} .
$$

\section{Prediction of $B P_{N D}$}

$B P_{N D}$ reflects the capacity of tissue for ligand-binding site interaction and includes terms for the target density $\left(B_{\max }\right.$, concentration of binding sites), the equilibrium dissociation rate constant $\left(K_{D}\right)$, and the free fraction in tissue $\left(f_{N D}\right)$. $B_{\max }$ measures the target concentration of binding sites in units of $\mathrm{fmol} / \mathrm{mg}$ of protein, fmol/mg of wet tissue, or $\mathrm{nM} . K_{D}$ is the reciprocal of the affinity of the ligand for the receptor in units of nM. At tracer dose, the in vivo $B P_{N D}$ is defined as:

$$
B P_{N D}=f_{N D} \frac{B_{\max }}{K_{D}}
$$

Thus, given the in vitro estimates of $B_{\max }, K_{D}$, and $f_{N D}$, we can predict the in vivo $B P_{N D}$ using Equation 8. Both $B_{\max }$ and $K_{D}$ can be derived from in vitro homogenate saturation binding measurements by fitting the saturation binding data to a Michaelis-Menten equation. $K_{i}$, derived from competitive binding experiments in which the binding of a single concentration of labeled ligand in the presence of various concentrations of unlabeled ligand is measured, can be used as an estimate for $K_{D}$ if the ligand and the blocker are the same. As the unit of $B_{\max }$ from the homogenate binding assay is generally in fmol/mg of protein, it can be converted to $\mathrm{nM}$ by assuming that the brain density is around $1 \mathrm{~g}$ wet weight $/ \mathrm{mL}$, and $1 \mathrm{fmol} / \mathrm{mg}$ of protein can be approximated 
by $0.05-0.1 \mathrm{fmol} / \mathrm{mg}$ of wet weight (13). In this way, we have refined our model for $B P_{N D}$ prediction as:

$$
B P_{N D}=f_{N D} \frac{B_{\max }^{H B}}{\alpha \cdot K_{D}},
$$

where $B_{\max }^{H B}$ is the $B_{\max }$ measured from homogenate binding assays in units of fmol/mg of protein, and $\alpha$ is the conversion coefficient from protein to tissue in units of milligram of protein per milliliter of tissue.

\section{Model Validation}

The In Silico/In Vitro/In Vivo Validation Dataset. The performance of our approach is evaluated on a dataset containing 28 compounds for which the in silico/in vitro and in vivo data exist (Table 1). For each compound, the in silico parameters such as $\operatorname{cog} D$ and $V x$ were obtained from in-house in silico models. For in vitro data, the $K_{i}$ of 23 of 28 molecules were measured in-house by competitive binding assays in human tissue at room temperature. The $K_{D}$ of $N$-methylspiperone (NMSP) and raclopride were measured in humans at $37^{\circ} \mathrm{C}$ and $23^{\circ} \mathrm{C}$, respectively (14). $K_{D}$ values of FLB-457, $R$-(-)-rolipram, and $S$ - $(+)$-rolipram were measured in rats at room temperature $(15,16)$. $B_{\max }$ values were obtained from the literature (9/10 targets) except for the $B_{\max }$ of GlyT1 in the human cortex, which was measured in-house using homogenate binding assays. Among those obtained from literature, the density of $5-\mathrm{HT}_{2 \mathrm{c}}$ was measured from rabbit homogenate (17), the density of PDE4 was from rat homogenate (18), and the density of the other targets was measured in human homogenate (19-24) (one other exception is that the $B_{\max }$ for $\mathrm{D}_{3}$ in human pallidum was determined using autoradiography $(25)$ in the absence of any homogenate binding data). Free fractions $f_{P}$ and $f_{N D}$ of all the molecules were measured in Yorkshire/Danish Landrace pig blood and brain tissue using equilibrium dialysis (12).

All 28 compounds were evaluated in preclinical PET studies in the Yorkshire/Danish Landrace pig $(\sim 40 \mathrm{~kg})$, at Aarhus University Hospital, in accordance with the Danish Animal Experimentation Act, under a license granted by the Danish Ministry of Justice. The animals were anesthetized by induction with ketamine and midazolam (both intramuscularly and intravenously) and maintained in deep anesthesia using isofluorane $(1 \%-2 \%$; Abbott) during scanning. Animals were placed supine in a Siemens ECAT EXACT HR tomograph (CTI), with the head immobilized in a custom-made head-holding device. For all scans, dynamic brain data were acquired in 3-dimensional mode for up to $90 \mathrm{~min}$ after the intravenous administration of the radiolabeled compound. Measured attenuation and scatter correction were applied, and the data were reconstructed using the reprojection algorithm (26). The resulting images had a spatial resolution of 5-7 $\mathrm{mm}$ in full width at half maximum (27). During the acquisition, $40 \times 2$ $\mathrm{mL}$ arterial blood samples were taken from the femoral artery to assay radioactivity in arterial plasma, and a subset of 17 were assayed for whole-blood radioactivity. Additional arterial samples $(6 \times 5 \mathrm{~mL})$ were drawn throughout the scan to determine the fraction of unmetabolized tracer in plasma by high-performance liquid chromatography and $\gamma$-counting. Integrated PET images were formed and used to rigidly register all the PET data to a Landrace pig brain atlas. Time-activity curves were generated for relevant regions of interest. Plasma parent input functions were generated by interpolating the discrete plasma data using linear interpolation and multiplying this by the continuous parent frac- tion derived from fitting the discrete metabolite data to an exponential approach to a constant. 1- and 2-tissue-compartmental kinetic analyses (28) were applied to the time-activity curves to derive regional estimates of the influx rate constant $\left(K_{I}\right)$ and total and nondisplaceable volumes of distribution $\left(V_{T}\right.$ and $\left.V_{N D}\right) . V_{N D}$ was obtained either from a reference region or from a blocked scan. The least-squares fitting procedure included a fixed 5\% blood volume and was performed with a Levenberg-Marquadt optimizer in Matlab (The MathWorks). The Akaike information criterion (29) was used as the model selection criterion to determine the most parsimonious kinetic model. $k_{2}$ was calculated as $K_{I} / V_{N D}$ and $B P_{N D}$ as $V_{T} / V_{N D}-1$.

Model Validation. First, the models to predict $K_{1}, k_{2}$, and $B P_{N D}$ were validated individually; second, the impact of the input function was considered; and finally, the overall in vivo predictive performance of the biomathematical approach was evaluated.

The $K_{l}$ values of the 28 compounds in the dataset were predicted using Equation 3, with permeability predicted using Equation 4 in conjunction with fixed values for perfusion $(0.6 \mathrm{~mL} /$ $\left.\mathrm{cm}^{3} / \min \right)(30)$ and capillary surface area $\left(150 \mathrm{~cm}^{2} / \mathrm{cm}^{3}\right.$ of brain) (12). As in vitro estimates of $K_{1}$ were expected to be lower because they were estimated from an in vitro MDCK cell line, it was necessary to introduce a scaling factor to account for global in vitro/in vivo $K_{l}$ differences of 3.43 (this was derived from a subset of the validation dataset $[n=13]$ for which there was high confidence of passive diffusion (31)). In vivo $K_{l}$ values of the full set of compounds were quantified from the PET data and compared with their predicted values.

$k_{2}$ values of the compounds were predicted using the predicted $K_{1}$ values, the $f_{P}$ and $f_{N D}$ estimates measured using equilibrium dialysis, and the apparent aqueous volumes in plasma and tissue $\left(V_{a q_{-} P}=0.98\right.$ solvent $/ \mathrm{mL}$ of plasma and $V_{a q_{-} T}=0.9$ solvent $/ \mathrm{mL}$ of tissue). In vivo $k_{2}$ values obtained from the PET data were compared with those predicted by the model.

Predicted $B P_{N D}$ estimates in the validation dataset were obtained using the $K_{D}$ or $K_{i}$ of the compound, their individual $f_{N D}$ measurement, and $B_{\max }$ from homogenate binding assays. To convert the unit of $B_{\max }$ from fmol/mg of protein to $\mathrm{nmol} / \mathrm{L}$, we assumed that there was 0.1 milligram of protein per milligram of wet weight ( $\alpha=10$ in Eq. 9). Predictions from in vitro assays were compared with the in vivo PET $B P_{N D}$ estimates.

The impact of the plasma input function was also evaluated. The $\% \operatorname{COV}\left[B P_{N D}\right]$ of the compounds in the dataset were calculated with 3 plasma input functions with different areas under the curve (AUCs), including a bolus input with fast clearance $\left(\mathrm{INP}_{1}\right.$, $\mathrm{AUC}_{0-90 \min }=131 \mathrm{kBq} \mathrm{min} / \mathrm{mL}$ ), a bolus input with moderate clearance $\left(\mathrm{INP}_{2}, \mathrm{AUC}_{0-90} \mathrm{~min}=264 \mathrm{kBq} \mathrm{min} / \mathrm{mL}\right)$, and a bolusplus-infusion input $\left(\mathrm{INP}_{3}, \mathrm{AUC}_{0-90 \mathrm{~min}}=367 \mathrm{kBq} \mathrm{min} / \mathrm{mL}\right)$. The in vivo PET $K_{l}, k_{2}$, and $B P_{N D}$ values of the compounds were used, and the $\% \operatorname{COV}\left[B P_{N D}\right]$ values were calculated for each input function.

Finally the overall performance of the modeling approach was assessed by estimating the $\% \operatorname{COV}\left[B P_{N D}\right]$ values using a standard input function $\left(\mathrm{INP}_{2}\right)$ and the $K_{1}, k_{2}$, and $B P_{N D}$ values predicted by Equations 3, 7, and 9.

\section{RESULTS}

\section{PET Data Analysis}

PET data for 4 of the 28 compounds are shown in Figure 2 , including examples displaying low brain penetration 
TABLE 1. Predicting In Vivo Performance of Radioligands from In Silico/In Vitro Data

\begin{tabular}{|c|c|c|c|c|c|c|c|c|c|c|c|c|}
\hline \multirow[b]{2}{*}{ Compound } & \multirow[b]{2}{*}{ Target } & \multirow[b]{2}{*}{$\mathrm{ROI}$} & \multicolumn{3}{|c|}{ In vitro measurement } & $\begin{array}{l}\text { In silico } \\
\text { properties }\end{array}$ & \multicolumn{2}{|c|}{$\begin{array}{l}\text { In vivo PET } \\
\text { data analysis }\end{array}$} & \multicolumn{4}{|c|}{$\begin{array}{l}\text { Biomathematical } \\
\text { model prediction }\end{array}$} \\
\hline & & & $f_{N D}$ & $B_{\max }$ & $K_{D}$ or $K_{i}$ & $\operatorname{clog} D$ & $V x \overline{K_{1}}$ & $B P_{N D}$ & $K_{1}$ & $k_{2}$ & $B P_{N D}$ & $\% \operatorname{COV}\left[B P_{N D}\right]$ \\
\hline${ }^{11} \mathrm{C}-\mathrm{GSK} 224558$ & $5-\mathrm{HT}_{6}$ & Striatum & 0.1230 .014 & $21.50(19)$ & 0.50 & 3.40 & 2.980 .400 .04 & 0.52 & 0.39 & 0.05 & 0.58 & $3.55 \%$ \\
\hline${ }^{11}$ C-GSK215083 & $5-\mathrm{HT}_{6}$ & Striatum & 0.2880 .039 & $21.50(19)$ & 1.59 & 2.90 & 2.750 .700 .06 & 1.05 & 0.51 & 0.08 & 0.53 & $3.06 \%$ \\
\hline${ }^{11} \mathrm{C}-$ GSK00000A & $5-\mathrm{HT}_{6}$ & Striatum & 0.2680 .040 & 21.50 & 0.49 & 1.00 & $2.790 .01 \mathrm{NQ}$ & NQ & 0.37 & 0.06 & 1.76 & $2.33 \%$ \\
\hline${ }^{11} \mathrm{C}-\mathrm{GSKO0000B}$ & $5-\mathrm{HT}_{6}$ & Striatum & 0.0100 .004 & $21.50(19)$ & 6.92 & 4.60 & 3.240 .160 .04 & 0.00 & 0.13 & 0.06 & 0.01 & $>100 \%$ \\
\hline${ }^{11} \mathrm{C}-\mathrm{GSK} 00000 \mathrm{C}$ & $5-\mathrm{HT}_{6}$ & Striatum & 0.0930 .004 & $21.50(19)$ & 0.63 & 1.40 & $2.750 .31 \mathrm{NQ}$ & NQ & 0.46 & 0.02 & 0.14 & $16.87 \%$ \\
\hline${ }^{11} \mathrm{C}-\mathrm{GSK} 00000 \mathrm{D}$ & NK1 & Striatum & 0.0040 .001 & $35.70(20)$ & 0.56 & 3.80 & 3.350 .160 .08 & 30.35 & 0.27 & 0.06 & 0.06 & $27.73 \%$ \\
\hline${ }^{11} \mathrm{C}-\mathrm{GSK} 00000 \mathrm{E}$ & NK1 & Striatum & 0.0100 .001 & $35.70(20)$ & 0.11 & 4.00 & 3.900 .090 .03 & 2.93 & 0.21 & 0.02 & 0.32 & $12.32 \%$ \\
\hline${ }^{11} \mathrm{C}-\mathrm{GR} 205171$ & NK1 & Striatum & 0.2990 .065 & 35.70 & 0.01 & 0.90 & 3.000 .320 .04 & 21.7 & 0.32 & 0.08 & 232 & $>100 \%$ \\
\hline${ }^{11} \mathrm{C}-\mathrm{GSK} 00000 \mathrm{~F}$ & NK1 & Striatum & 0.0260 .001 & $35.70(20)$ & 0.79 & 2.20 & 3.060 .330 .05 & 0.50 & 0.52 & 0.02 & 0.05 & $34.09 \%$ \\
\hline${ }^{18} \mathrm{~F}-\mathrm{GSK} 00000 \mathrm{G}$ & NK1 & Striatum & 0.0270 .011 & $35.70(20)$ & 1995 & 4.90 & $3.520 .00 \mathrm{NQ}$ & NQ & 0.08 & 0.03 & 0.00 & $>100 \%$ \\
\hline${ }^{11} \mathrm{C}-\mathrm{GSKOOOOOH}$ & NK1 & Striatum & 0.0590 .010 & 35.70 & 0.50 & 3.90 & 3.490 .220 .03 & 31.30 & 0.24 & 0.04 & 0.70 & $4.37 \%$ \\
\hline${ }^{11} \mathrm{C}-\mathrm{GSK} 00000 \mathrm{I}$ & NK1 & Striatum & 0.0130 .001 & $35.70(20)$ & 0.14 & 3.90 & 3.490 .350 .04 & 0.49 & 0.24 & 0.03 & 0.35 & $7.67 \%$ \\
\hline${ }^{11} \mathrm{C}-\mathrm{GSK} 00000 \mathrm{~J}$ & NK1 & Striatum & 0.0730 .003 & 35.70 & 0.96 & 3.60 & 3.340 .360 .02 & 0.89 & 0.32 & 0.01 & 0.11 & $>100 \%$ \\
\hline${ }^{11} \mathrm{C}-\mathrm{R}-(-)$-rolipram & PDE4 & Frontal ctx & 0.2580 .192 & $20.60^{*}(18)$ & $1.76^{\star}(16)$ & 1.40 & 2.140 .450 .26 & 5.33 & 0.55 & 0.45 & 2.25 & $2.30 \%$ \\
\hline${ }^{11} \mathrm{C}-\mathrm{S}-(+)$-rolipram & PDE4 & Frontal ctx & 0.3000 .194 & $20.60^{*}(18)$ & $12.4^{*}(16)$ & 1.40 & 2.140 .200 .07 & 0.75 & 0.55 & 0.39 & 0.32 & $6.95 \%$ \\
\hline${ }^{11} \mathrm{C}-\mathrm{GSK} 00000 \mathrm{~K}$ & $D_{3}$ & Pallidum & 0.3270 .147 & $3.16^{+}(25)$ & 5.37 & 2.20 & 2.570 .720 .13 & 0.26 & 0.58 & 0.29 & 0.09 & $18.11 \%$ \\
\hline${ }^{11} \mathrm{C}$-flumazenil & $\mathrm{BDZ}$ & Frontal ctx & 0.6300 .553 & 70.00 & 2.20 & 0.90 & 2.090 .480 .43 & 5.96 & 0.43 & 0.41 & 17.6 & $2.02 \%$ \\
\hline${ }^{11} \mathrm{C}-\mathrm{GSK} 00000 \mathrm{~L}$ & CRF1 & Striatum & 0.0360 .012 & 17.05 & 64.6 & 0.90 & 2.990 .670 .08 & NQ & 0.32 & 0.12 & 0.00 & $>100 \%$ \\
\hline${ }^{11} \mathrm{C}-\mathrm{NMSP}$ & $\mathrm{D}_{2}$ & Striatum & 0.1580 .048 & 13.00 & $0.22^{\ddagger}(14)$ & 1.00 & 3.150 .440 .13 & 315.4 & 0.33 & 0.11 & 2.86 & $1.93 \%$ \\
\hline${ }^{11} \mathrm{C}-\mathrm{NMSP}$ & $\mathrm{D}_{2}$ & Thalamus & 0.1580 .048 & $0.91(23)$ & $0.22^{\ddagger}(14)$ & 1.00 & 3.150 .320 .09 & 0.45 & 0.33 & 0.11 & 0.20 & $8.09 \%$ \\
\hline${ }^{11} \mathrm{C}-\mathrm{FLB}-457$ & $\mathrm{D}_{2}$ & Striatum & 0.4760 .211 & $13.00(21)$ & $0.02^{*}(15)$ & 0.80 & 2.520 .680 .22 & 9.29 & 0.34 & 0.17 & 137 & $27.75 \%$ \\
\hline${ }^{11} \mathrm{C}-\mathrm{FLB}-457$ & $\mathrm{D}_{2}$ & Thalamus & 0.4760 .211 & $0.91(23)$ & $0.02^{*}(15)$ & 0.80 & 2.520 .660 .21 & 0.87 & 0.34 & 0.17 & 9.62 & $2.23 \%$ \\
\hline${ }^{11} \mathrm{C}$-raclopride & $\mathrm{D}_{2}$ & Striatum & 0.1510 .134 & $13.00(21)$ & $0.85(14)$ & 1.60 & 2.450 .460 .41 & 1.88 & 0.54 & 0.52 & 2.05 & $2.55 \%$ \\
\hline${ }^{11} \mathrm{C}$-raclopride & $\mathrm{D}_{2}$ & Thalamus & 0.1510 .134 & $0.91(23)$ & $0.85(14)$ & 1.60 & 2.450 .450 .40 & 0.40 & 0.54 & 0.52 & 0.14 & $15.80 \%$ \\
\hline${ }^{11} \mathrm{C}-\mathrm{GSK} 00000 \mathrm{M}$ & GlyT1 & Ctx & 0.0090 .101 & 50.00 & 3.09 & 2.70 & $\begin{array}{lll}3.21 & 0.02 & 0.03\end{array}$ & 0.56 & 0.48 & 5.75 & 1.63 & $8.69 \%$ \\
\hline${ }^{11}$ C-GSK931145 & GlyT1 & Ctx & 0.2790 .100 & 50.00 & 1.51 & 3.80 & 2.980 .070 .07 & 0.62 & 0.30 & 0.12 & 3.31 & $2.11 \%$ \\
\hline${ }^{11} \mathrm{C}-\mathrm{GSK} 00000 \mathrm{~N}$ & GlyT1 & Ctx & 0.0240 .041 & 50.00 & 5.75 & 4.70 & 3.290 .030 .02 & 0.00 & 0.11 & 0.20 & 0.44 & $9.31 \%$ \\
\hline${ }^{11} \mathrm{C}-\mathrm{GSK} 000000$ & GlyT1 & Ctx & 0.1290 .016 & 50.00 & 1.05 & 3.60 & 2.870 .190 .03 & 30.07 & 0.36 & 0.05 & 0.76 & $2.93 \%$ \\
\hline${ }^{11} \mathrm{C}-\mathrm{PK} 11195$ & PBZR & Frontal ctx & 0.0660 .022 & 32.20 & 4.30 & 4.60 & 2.740 .330 .05 & 0.55 & 0.14 & 0.05 & 0.17 & $13.34 \%$ \\
\hline${ }^{11} \mathrm{C}-\mathrm{GSK} 00000 \mathrm{P}$ & $5-\mathrm{HT}_{2 \mathrm{C}}$ & Frontal ctx & 0.0670 .009 & $3.70^{\S}(17)$ & 0.31 & 1.90 & 3.550 .080 .03 & 0.28 & 0.45 & 0.07 & 0.11 & $10.60 \%$ \\
\hline${ }^{11} \mathrm{C}-\mathrm{GSK} 00000 \mathrm{Q}$ & $5-\mathrm{HT}_{2 \mathrm{C}}$ & Frontal ctx & 0.1380 .010 & $3.70^{\S}(17)$ & 0.83 & 1.90 & 3.650 .090 .01 & 0.65 & 0.44 & 0.04 & 0.05 & $24.53 \%$ \\
\hline $\begin{array}{r}{ }^{\star} \text { Measured in rat. } \\
{ }^{\dagger} \text { Measured using } \\
{ }^{\ddagger} \text { Measured at } 37^{\circ} \\
{ }^{\S} \text { Measured in rabl } \\
f_{P} \text { and } f_{N D} \text { were } \mathrm{m} \\
\text { binding assays at roc } \\
\text { for } B_{\text {max }}, K_{D} \text {, and } K_{i} \text { a } \\
B P_{N D} \text { are unitless. N }\end{array}$ & $\begin{array}{l}\text { autora } \\
\text { C. } \\
\text { bit. } \\
\text { easure } \\
\text { om tem } \\
\text { are nM; }\end{array}$ & $\begin{array}{l}\text { in Yorkshi } \\
\text { erature in } \\
\text { unit for } V x\end{array}$ & $\begin{array}{l}\text { e/Danish } \mathrm{La} \\
\text { רuman tissu } \\
\mathrm{cm}^{3} \mathrm{~mol}^{-1}\end{array}$ & $\begin{array}{l}\text { arace pig } \\
\text { unless ot } \\
\text { D0; units } \\
\text { ences. R }\end{array}$ & wise $\mathrm{no}$ & (In & $\begin{array}{l}\text { alysis. } B_{\max }, K_{L} \\
\text { v. } \mathrm{NQ} \text { denotes } \\
/ \mathrm{cm}^{3} / \mathrm{min} \text { and } \\
\text { est; } \mathrm{Ctx}=\mathrm{cor}\end{array}$ & $\begin{array}{l}\text {, or } K_{i} \\
\text { honqu } \\
\text { nin }{ }^{-1} \text {, } \\
\text { ex. }\end{array}$ & iWe & $m$ & vi & $\begin{array}{l}\text { homogena } \\
\text { stimate. Un } \\
{ }_{D}, c \log D, \text { a }\end{array}$ \\
\hline
\end{tabular}

(Fig. 2B), high nonspecific binding (Fig. 2C), slow kinetics (Fig. 2D), and good specific binding and kinetics (Fig. 2E). The in vivo $K_{1}, k_{2}$, and $B P_{N D}$ values estimated from the PET data for all 28 compounds are listed in Table 1.

\section{Validation of $K_{1}$ Prediction Model}

Scatter plots for the predicted and measured in vivo $K_{1}$ are shown in Figure 3. The Pearson correlation coefficient was $r=0.402(P=0.025)$ and reflected a reasonably good correlation when considering the complexity of predicting in vivo brain penetration.

\section{Validation of $k_{2}$ Prediction Model}

Scatter plots for the predicted and measured in vivo $k_{2}$ values are shown in Figure 4. There was good correlation between the predicted and the in vivo $k_{2}(r=0.85, P<$
0.0001). Three compounds that had nonquantifiable in vivo $k_{2}$ (mostly because of low brain penetration) and 1 compound that had a large predicted $k_{2}$ value $\left(k_{2}=5.75\right)$ driven by a small $f_{p}$ (Table 1 ) were omitted.

\section{Validation of $B P_{N D}$ Prediction Model}

Scatter plots for the predicted and measured in vivo $B P_{N D}$ are given in Figure 5. $\mathrm{D}_{3}$ and CRF1 data are omitted from the plot because $B_{\max }$ for $\mathrm{D}_{3}$ was measured using autoradiography, and the in vivo $B P_{N D}$ for CRF1 was not quantifiable (Table 1). Because $B P_{N D}$ estimates of ligands can vary by 3 orders of magnitude, they are plotted using a $\log$ scale. It shows that $B P_{N D}$ values predicted from in vitro $B_{\max }, K_{D}$ or $K_{i}$ data are highly correlated with the measured in vivo $B P_{N D}(r=0.822, P<0.0001)$. 

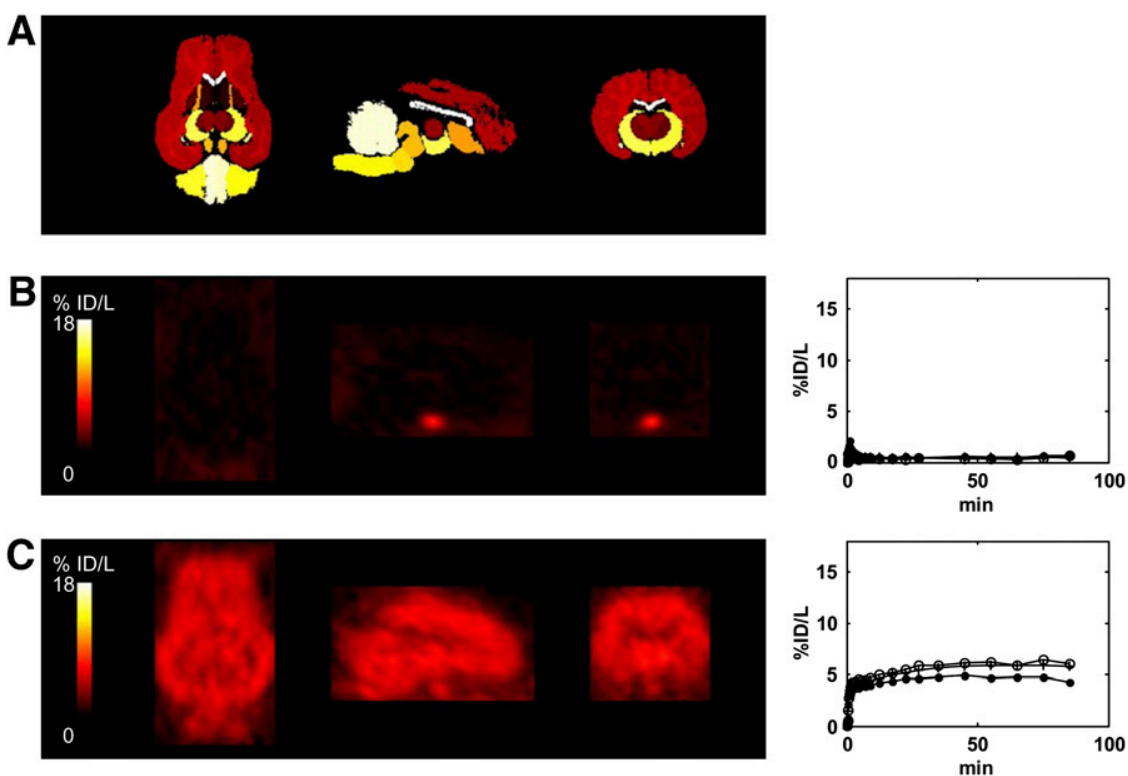

FIGURE 2. Coregistered Landrace pig brain atlas and PET images (transverse, coronal, and sagittal slices), with corresponding time-activity curves for striatum (O), frontal cortex (+), and cerebellum $(0)$ for 4 candidate CNS radioligands. (A) Landrace pig brain atlas. (B) ${ }^{11} \mathrm{C}$-GSK00000A targeting serotonin $5-\mathrm{HT}_{6}$ receptor. (C) ${ }^{11} \mathrm{C}-$ GSK00000D targeting NK1 receptor. (D) ${ }^{11} \mathrm{C}-\mathrm{GR} 205171$ targeting NK1 receptor. (E) ${ }^{11} \mathrm{C}$-raclopride targeting $\mathrm{D}_{2}$ receptor. PET images are integral images over $90 \mathrm{~min}$. $\% \mathrm{ID} / \mathrm{L}=$ percentage injected dose per liter.
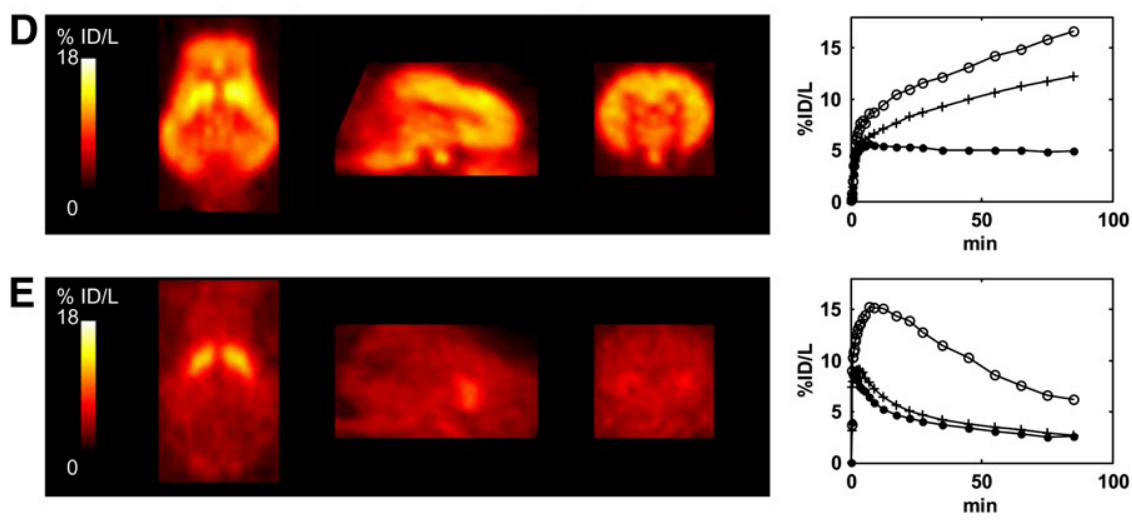

\section{Evaluation of Impact of Input Functions}

The impact of 3 different plasma input functions on the $\% C O V\left[B P_{N D}\right]$ estimates was assessed. For all compounds in the dataset, increased plasma clearance rates decreased $\% \operatorname{COV}\left[B P_{N D}\right]$ estimates (data not shown), which is consistent with faster kinetics yielding better quantification results. However, the magnitude of this effect was small, with values proportionally changing by less than $24 \%$ when the input function with the fastest clearance rate was compared with the bolus-plus-infusion input.

FIGURE 3. Relationship between predicted $K_{1}$ values and those measured in vivo for validation dataset $(r=0.402, P=0.025)$. Line of identity is shown.

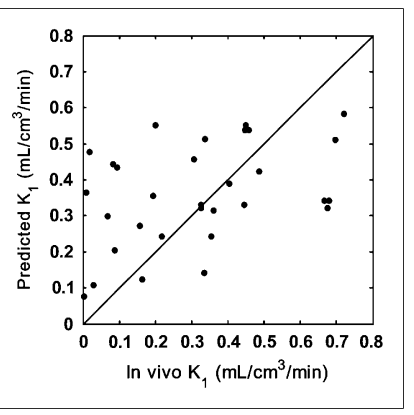

\section{Overall Prediction of In Vivo Performance}

The predicted \%COV $\left[B P_{N D}\right]$ values of 28 compounds using a standard input function are given in Table 1 . The in vivo performance prediction shows that widely accepted good ligands such as ${ }^{11} \mathrm{C}$-flumazenil and ${ }^{11} \mathrm{C}$-raclopride had small $\% \operatorname{COV}\left[B P_{N D}\right]$ values of $2.02 \%$ and $2.55 \%$, respectively, whereas poor imaging probes were identified by a higher \% COV $\left[B P_{N D}\right]$ such as ${ }^{11} \mathrm{C}-(R)-\mathrm{PK} 11195(13.34 \%$, low signal) and ${ }^{11} \mathrm{C}-\mathrm{GR} 205171(>100 \%$, near-irreversible kinetics). For targets with multiple regions of interest at varying densities, the model predicts the performance of the radioligand for each region. For example, ${ }^{11}$ C-FLB457 was rated by the model as a good $\mathrm{D}_{2}$ ligand in the thalamus $(2.23 \%)$ but a poor $\mathrm{D}_{2}$ ligand in striatum $(27.75 \%)$. Within a specific target, the model was able to rank the candidates according to their \% COV $\left[B P_{N D}\right]$ values, and the results were generally consistent with the historical decisions made by scientists after evaluation of the in vivo PET data of all the candidate compounds. For example, of 4 candidates for the GlyT1 transporter, the model selected ${ }^{11} \mathrm{C}$-GSK931145 $(2.11 \%)$ as the best ligand. 


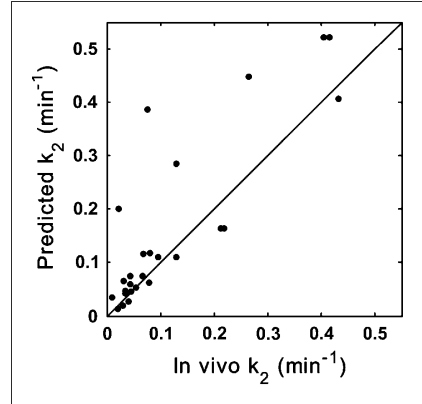

FIGURE 4. Relationship between predicted $k_{2}$ values and those measured in vivo for validation dataset $(r=0.85, P<0.0001)$. Line of identity is shown.

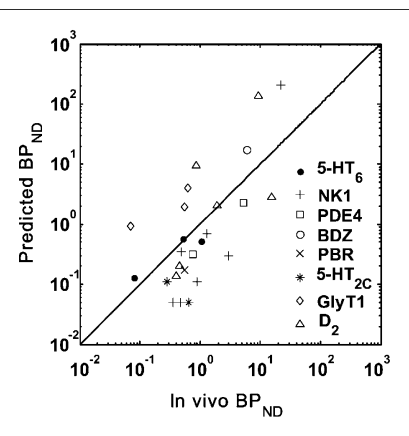

FIGURE 5. Relationship between predicted $B P_{N D}$ values and those measured in vivo for 8 targets in validation dataset $(r=0.822$, $P<0.0001$ ). Line of identity is shown.

\section{DISCUSSION}

Motivated by the limitations of current approaches to radioligand discovery and development, we have introduced a biomathematical modeling approach that uses in silico/in vitro data of candidate molecules to predict their in vivo performance. The model incorporates components accounting for BBB penetration, specific binding, nonspecific binding, and kinetics and derives a relevant outcome metric (\% COV $\left.\left[B P_{N D}\right]\right)$ from Monte Carlo simulations as a measure of in vivo performance. This approach is able to select promising ligands from a set of candidates, consistent with the decisions made by scientists after the evaluation of in vivo PET data. In addition, the ranking of different radioligands across targets is generally consistent with their known performance; that is, ${ }^{11} \mathrm{C}$-flumazenil and ${ }^{11} \mathrm{C}$-raclopride are ideal whereas ${ }^{11} \mathrm{C}-(R)$-PK11195 and ${ }^{11} \mathrm{C}-\mathrm{GR} 205171$ are not.

Nevertheless, given the complexity of radioligand design and in vivo biological systems, there are several limitations to our current model. For example, one of the weaknesses is that it assumes passive diffusion across the BBB. However, it is known that the endothelial membranes of the BBB contain several transport proteins that can actively transport compounds into the brain (e.g., transporters for amino acids and monocarboxylic acids) or efflux compounds from the brain (e.g., P-glycoprotein). Although several permeability models have been developed over the past few decades $(10,32-34)$, none was able to accurately predict permeabil- ity for all the transport mechanisms because of the complexity of these active and facilitated transport systems in the BBB. We are aware that in our validation dataset there exist several compounds that are likely to cross the BBB actively, and these have been identified in a recent study combining equilibrium dialysis assays with in vivo PET data (31). To investigate this further, we excluded those compounds predicted to have active transport mechanisms and reassessed the correlation between the predicted and measured in vivo $K_{l}$. The result showed that the correlation coefficient improved from 0.40 to 0.59 (Fig. 6A). Thus, if it were possible to add a model to predict active and passive transport, then the overall performance could be improved further.

Similarly, because the prediction of $k_{2}$ was based on the predicted $K_{1}$ values under the assumption of passive diffusion, the correlation coefficient between the predicted and measured in vivo $k_{2}$ was also improved from 0.85 to 0.93 (Fig. 6B) when we excluded those compounds predicted to have active transport mechanisms. In the validation of the model to predict $k_{2}$, free fractions in plasma and tissue were measured using pig homogenate to be consistent with the in vivo PET data from pigs. Although the free fraction in tissue is generally constant across species (35), the free fraction in plasma can vary widely.

Species differences should be carefully considered, particularly in the prediction of $B P_{N D}$. Most of the homogenate-binding $B_{\max }, K_{D}$, or $K_{i}$ data used in our validation
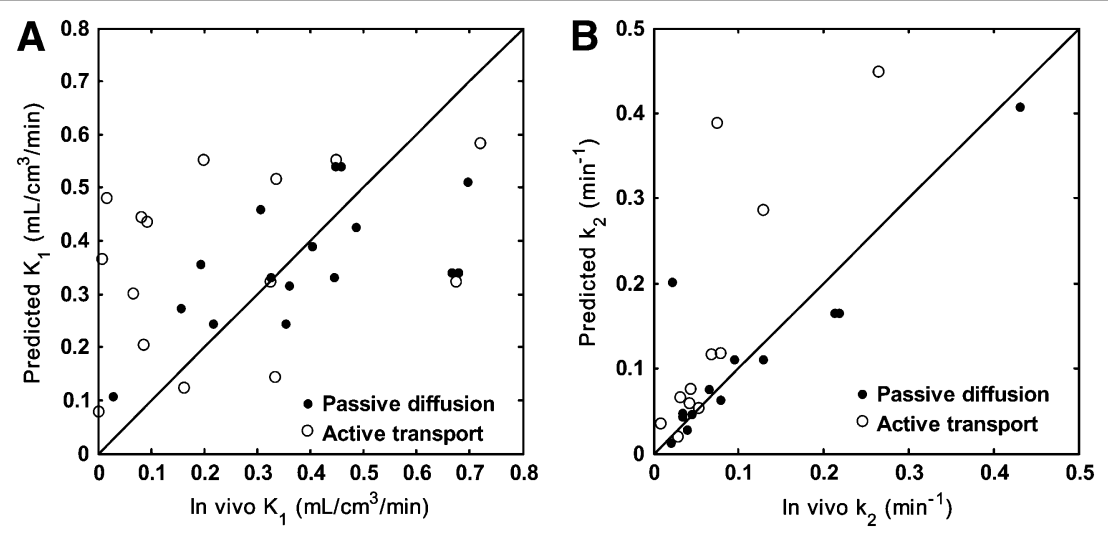

FIGURE 6. Relationship between predicted and measured in vivo data partitioned according to likely passive diffusion and active transport status. (A) Relationship between predicted $K_{1}$ values and those measured in vivo $(r=$ $0.59, P=0.017$, active transport compounds excluded from correlation). (B) Relationship between predicted $k_{2}$ values and those measured in vivo $(r=$ $0.93, P<0.0001$, active transport compounds excluded from correlation). Line of identity is shown. 
dataset were obtained in humans rather than in pigs, and these parameters may vary across species. Different in vitro assay conditions may also cause discrepancies between the predicted $B P_{N D}$ values and those measured in vivo. First, the binding of ligands to their targets can be a temperaturedependent process, and ideally the incubation should be conducted at body temperature $\left(37^{\circ} \mathrm{C}\right)$. In our validation dataset, however, the $K_{i}$ values of most of the compounds were measured in human tissue at room temperature, which may lead to some discrepancy. Second, the difference in buffer may affect the final estimation as well. A physiological buffer (e.g., Tris- $\mathrm{HCl}$ or $\mathrm{N}$-(2-hydroxyethyl)piperazine- $N^{\prime}$-(2-ethanesulfonic acid) buffer at $\mathrm{pH}$ 7.4, with high concentration of $\mathrm{Na}^{+}$) should ideally be used to approximate the in vivo conditions. In addition, target density and affinity measured in the in vitro open cell environment using homogenate binding assays may be different from those estimated in vivo because of the complex in vivo environment that may involve target internalization and endogenous competition (e.g., $\mathrm{D}_{2}$ receptor (36)). Thus, care should be taken when using in vitro estimates to quantify the in vivo situation. For example, as shown in Table 1, there is a discrepancy between the prediction of NMSP in the striatum and actual in vivo experience. The predicted $B P_{N D}$ value (2.86) is smaller than the in vivo value (15.4). This discrepancy could be explained by an underestimation of the affinity or the tissue free fraction. Another potential discrepancy between some of the predicted $B P_{N D}$ values and those measured in vivo is due to the conversion coefficient from protein to wet weight $\alpha$, which was assumed to be 10 .

In addition, in our validation only the impact of clearance of the input function was considered, which was shown not to be critical on the overall outcome measure $B P_{N D}$ and its associated performance metric (\%COV[BP $\left.\left.{ }_{N D}\right]\right)$. Nevertheless, there are several factors that will lead to different plasma concentrations, and the ability to predict the plasma time course would further improve the accuracy of the biomathematical model. Prediction of the time course would require a prediction of the dosimetry profile and metabolism. Although predicting full dosimetry may be difficult, it may be possible to get some idea about metabolism through in silico/in vitro assays involving liver microsomes, for those compounds metabolized via the liver. The ability to predict metabolism has further consequences for the development of CNS radioligands because the production of radiolabeled lipophilic products that enter the brain may compromise radioligand quantification. Thus, if it were possible to screen out compounds that produce lipophilic metabolites, it would further reduce attrition in the development process.

Another limitation of our current approach is the lack of explicit selectivity screening. The model could easily be extended to take additional affinity (and associated $B_{\max }$ ) data from other targets to accordingly account for the selectivity of the radioligand. Finally, it would also be useful to capture information on radiolabeling feasibility of candidate molecules and include this in the overall prediction of success.

The approach discussed here has focused on CNS radioligand discovery and development. Nevertheless, this biomathematical framework could be generalized to develop radiotracers for disease understanding, diagnosis, and monitoring of therapies in other organs in addition to the brain. Appropriate modifications would necessitate an understanding of the underlying biological system and the desired outcome measure. For example, the development of a radiotracer for oncology applications in the body may require additional components to screen for metabolites and could choose an irreversible uptake rate constant as the outcome measure of interest.

\section{CONCLUSION}

A biomathematical modeling approach to CNS radioligand discovery and development has the potential to predict the in vivo performance of ligands from in silico/in vitro data before any in vivo work. This would enable us to target the right molecules for radiolabeling, efficiently leverage large compound databases, and significantly reduce the attrition in the development of successful CNS imaging probes.

\section{ACKNOWLEDGMENTS}

We thank Laurent Martarello and Jan Passchier for many interesting and insightful discussions around radiochemistry, Cristian Salinas for guidance on the analysis of preclinical PET data, Graham Searle and Andri Tziortzi for discussions around data analysis, Mats Bergstrom and Christine Parker for discussions around in vitro assays of radioligand binding, Scott Summerfield for provision of the equilibrium dialysis data, Hugh Herdon for provision of GlyT1 data, and the Aarhus PET Centre staff for helping in the acquisition of all the in vivo PET data over a number of years of excellent collaboration. Software for performing the methodology presented in this paper may be freely obtained, for academic purposes, by emailing the author: qiguo0508@gmail.com. A portion of this work was funded by Biotechnology and Biological Sciences Research Council (U.K.) and GlaxoSmithKline.

\section{REFERENCES}

1. Laruelle M, Slifstein M, Huang Y. Relationships between radiotracer properties and image quality in molecular imaging of the brain with positron emission tomography. Mol Imaging Biol. 2003;5:363-375.

2. van de Waterbeemd H, Camenisch G, Folkers G, Chretien J, Raevsky O. Estimation of blood-brain barrier crossing of drugs using molecular size and shape, and H-bonding descriptors. J Drug Target. 1998;6:151-165.

3. Patel S, Ndubizu O, Hamill T, et al. Screening cascade and development of potential positron emission tomography radiotracers for mGluR5: in vitro and in vivo characterization. Mol Imaging Biol. 2005;7:314-323.

4. Wong DF, Pomper MG. Predicting the success of a radiopharmaceutical for in vivo imaging of central nervous system neuroreceptor systems. Mol Imaging Biol. 2003;5:350-362. 
5. Fowler JS. Radiotracer chemistry in 2003: better prediction needed. J Nucl Med. 2004;45(2): $15 \mathrm{~N}-58 \mathrm{~N}$.

6. Innis RB, Cunningham VJ, Delforge J, et al. Consensus nomenclature for in vivo imaging of reversibly binding radioligands. J Cereb Blood Flow Metab. 2007;27:1533-1539.

7. Crone C. The permeability of capillaries in various organs as determined by use of the indicator diffusion method. Acta Physiol Scand. 1963;58:292-305.

8. Renkin EM. Transport of potassium-42 from blood to tissue in isolated mammalian skeletal muscles. Am J Physiol. 1959;197:1205-1210.

9. Gross PM, Sposito NM, Pettersen SE, Fenstermacher JD. Differences in function and structure of the capillary endothelium in gray matter, white matter and a circumventricular organ of rat brain. Blood Vessels. 1986;23:261-270.

10. Lanevskij K, Japertas P, Didziapetris R, Petrauskas A. Ionization-specific prediction of blood-brain permeability. J Pharm Sci. 2009;98:122-134.

11. Zhao YH, Abraham MH, Zissimos AM. Determination of McGowan volumes for ions and correlation with van der Waals volumes. J Chem Inf Comput Sci. 2003;43:1848-1854.

12. Summerfield SG, Read K, Begley DJ, et al. Central nervous system drug disposition: the relationship between in situ brain permeability and brain free fraction. J Pharmacol Exp Ther. 2007;322:205-213.

13. Backstrom I, Bergstrom M, Marcusson J. High affinity $\left[{ }^{3} \mathrm{H}\right]$ paroxetine binding to serotonin uptake sites in human brain tissue. Brain Res. 1989;486:261-268.

14. Hall H, Wedel I, Halldin C, Kopp J, Farde L. Comparison of the in vitro receptor binding properties of $N-\left[{ }^{3} \mathrm{H}\right]$ methylspiperone and $\left[{ }^{3} \mathrm{H}\right]$ raclopride to rat and human brain membranes. J Neurochem. 1990;55:2048-2057.

15. Halldin C, Farde L, Hogberg T, et al. Carbon-11-FLB 457: a radioligand for extrastriatal $\mathrm{D}_{2}$ dopamine receptors. J Nucl Med. 1995;36:1275-1281.

16. Parker CA, Matthews JC, Gunn RN, et al. Behaviour of $\left[{ }^{11} \mathrm{C}\right] R(-)$ - and $\left[{ }^{11} \mathrm{C}\right] S(+)$-rolipram in vitro and in vivo, and their use as PET radiotracers for the quantificative assay of PDE4. Synapse. 2005;55:270-279.

17. Aloyo VJ, Harvey JA. Antagonist binding at $5-\mathrm{HT}_{2 \mathrm{~A}}$ and $5-\mathrm{HT}_{2 \mathrm{C}}$ receptors in the rabbit: high correlation with the profile for the human receptors. Eur $J$ Pharmacol. 2000;406:163-169.

18. Schneider HH, Schmiechen R, Brezinski M, Seidler J. Stereospecific binding of the antidepressant rolipram to brain protein structures. Eur J Pharmacol. 1986;127:105-115.

19. Hirst WD, Minton JAL, Bromidge SM, et al. Characterization of $\left[{ }^{125} \mathrm{I}\right]-\mathrm{SB}-$ 258585 binding to human recombinant and native $5-\mathrm{HT}_{6}$ receptors in rat, pig and human brain tissue. Br J Pharmacol. 2000;130:1597-1605.

20. Griffante C, Carletti R, Andreetta F, Corsi M. [ $\left.{ }^{3} \mathrm{H}\right] \mathrm{GR} 205171$ displays similar NK1 receptor binding profile in gerbil and human brain. $\mathrm{Br} J$ Pharmacol. 2006;148:39-45.

21. Hall H, Farde L, Sedvall G. Human dopamine receptor subtypes: in vitro binding analysis using ${ }^{3} \mathrm{H}-\mathrm{SCH} 23390$ and ${ }^{3} \mathrm{H}$-raclopride. J Neural Transm. 1988;73:7-21.
22. Hall H, Litton JE, Halldin C, Kopp J, Sedvall G. Studies on the binding of $\left[{ }^{3} \mathrm{H}\right]$ flumazenil and $\left[{ }^{3} \mathrm{H}\right]$ sarmazenil in post-mortem human brain. Hum Psychopharmacol. 1992;7:367-377.

23. Kessler RM, Whetsell WO, Ansari MS, et al. Identification of extrastriatal dopamine $\mathrm{D}_{2}$ receptors in post mortem human brain with $\left[{ }^{125} \mathrm{I}\right]$ epidepride. Brain Res. 1993;23:237-243.

24. Sullivan GM, Parsey RV, Kumar JSD, et al. PET Imaging of CRF1 with $\left[{ }^{11} \mathrm{C}\right] \mathrm{R} 121920$ and $\left[{ }^{11} \mathrm{C}\right] \mathrm{DMP} 696$ : is the target of sufficient density? Nucl Med Biol. 2007;34:353-361.

25. Murray AM, Ryoo HL, Gurevich E, Joyce JN. Localization of dopamine $\mathrm{D}_{3}$ receptors to mesolimbic and $\mathrm{D}_{2}$ receptors to mesostriatal regions of human forebrain. Proc Natl Acad Sci USA. 1994;91:11271-11275.

26. Kinahan PE, Rogers JG. Analytic 3D image reconstruction using all detected events. IEEE Trans Nucl Sci. 1989;36:964-968.

27. Wienhard K, Dahlbom M, Eriksson L, et al. The ECAT EXACT HR: performance of a new high resolution positron scanner. J Comput Assist Tomogr. 1994;18:110-118.

28. Gunn RN, Gunn SR, Cunningham VJ. Positron emission tomography compartmental models. J Cereb Blood Flow Metab. 2001;21:635-652.

29. Akaike H. A new look at statistical model identification. IEEE Trans Automat Contr. 1974;19:716-723.

30. Cumming P, Rosa-Neto P, Watanabe H, et al. Effects of acute nicotine on hemodynamics and binding of $\left[{ }^{11} \mathrm{C}\right]$ raclopride to dopamine $\mathrm{D}_{2,3}$ receptors in pig brain. Neuroimage. 2003;19:1127-1136.

31. Gunn RN, Summerfield SG, Salinas CA, et al. Combining PET and equilibrium dialysis to assess blood brain barrier transport. Paper presented at: Brain'07 and BrainPET'07; May 20-24, 2007; Osaka, Japan.

32. Levin VA. Relationship of octanol/water partition coefficient and molecular weight to rat brain capillary permeability. J Med Chem. 1980;23:682684.

33. Liu X, Tu M, Kelly RS, Chen C, Smith BJ. Development of a computational approach to predict blood-brain barrier permeability. Drug Metab Dispos. 2004;32:132-139.

34. Abraham MH. The factors that influence permeation across the blood-brain barrier. Eur J Med Chem. 2004;39:235-240.

35. Summerfield SG, Lucas AJ, Porter RA, et al. Toward an improved prediction of human in vivo brain penetration. Xenobiotica. 2008;38:1518-1535.

36. Sun W, Ginovart N, Ko F, Seeman P, Kapur S. In vivo evidence for dopaminemediated internalization of $\mathrm{d}_{2}$-receptors after amphetamine: differential findings with $\left[{ }^{3} \mathrm{H}\right]$ raclopride versus $\left[{ }^{3} \mathrm{H}\right]$ spiperone. Mol Pharmacol. 2003;63: 456-462.

37. Rao VLR, Butterworth RF. Characterization of binding sites for the $\omega 3$ receptor ligands $\left[{ }^{3} \mathrm{H}\right] \mathrm{PK} 11195$ and $\left[{ }^{3} \mathrm{H}\right] \mathrm{RO} 5-4864$ in human brain. Eur $J$ Pharmacol. 1997;340:89-99. 